Part of Journal of Research of the National Bureau of Standards, Volume 25, July 1940

\title{
A VACUUM-TUBE ALTERNATING-VOLTAGE COMPENSATOR
}

\author{
By Irvin L. Cooter, Frank Wenner, and Chester Peterson
}

\begin{abstract}
A vacuum-tube amplifier of the type described is a simple and inexpensive means for compensating small alternating voltages. Two such amplifiers and an iron-cored inductor with air gaps are used as a choke in a circuit having a resistance of approximately $20 \mathrm{ohms}$ and in which there is a direct current of approximately 1 ampere. The effectiveness of the arrangement is such that rapid variations of the potential drop amounting to 0.1 volt in a part of the circuit result in variations in the current of less than 1 microampere.
\end{abstract}

One of the methods now being used in this Bureau in an absolute measurement of electrical resistance requires that the test current through a standard resistor be kept very nearly constant over successive time intervals of a few hundredths of a second. This requirement must be met even though, in the same circuit, there are rapid variations of resistances and voltages of sufficient magnitude to disturb the constancy of the current unless their effects are nullified. The purpose of this paper is to describe briefly the means employed to hold the current constant.

The problem may be visualized by referring to figure 1 .

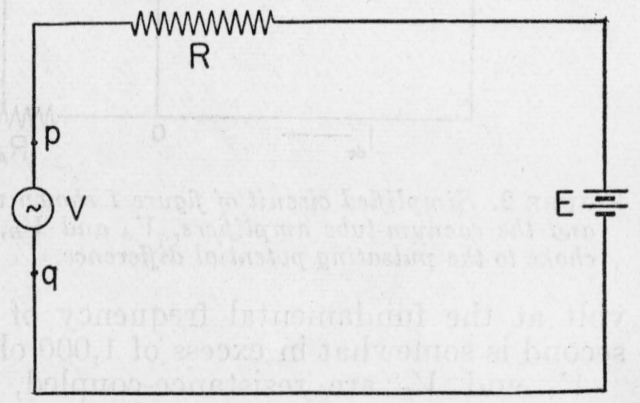

FIGURE 1. Simplified circuit containing a resistor, $R$, in which it is necessary to maintain a direct current which, over short intervals of time, is constant to one part in a million while there is a pulsating potential difference between $p$ and, $q$.

In this figure, $E$ represents a battery supplying direct current of approximately 1 ampere through the 1 -ohm resistor, $R$. For the purpose of measurement, it is necessary that the fluctuations of current in $R$ should be less than 1 microampere from its average value over successive time intervals of a few hundredths of a second. However, the operation of a rotating commutator and other parts connected to $p$ and $q$, but not shown in figure 1, constitutes in effect a source of alternating voltage, $V$, between the points $p$ and $q$. Although this alternating voltage has a fundamental frequency of approximately 45 cycles per second, 
the wave shape is nonsine and not strictly the same in successive cycles. The mechanisms connected to $p$ and $q$ are so constructed and adjusted that peak values of this fluctuating voltage do not exceed 0.1 volt.

In a problem such as is stated above, an iron-cored inductor with suitable air gaps in the magnetic circuit constitutes one of the simplest and most effective known means for reducing the amplitude of pulsations in the current. An inductor of this type could readily be made to reduce the amplitude of the current pulsations to one part in ten thousand, but not to one part in a million of the average current. Consequently it was necessary to devise means for supplementing the inductor.

The solution to our problem, found after considerable experimentation, is shown in figure 2. In figure $2, L$ is an iron-cored inductor having a resistance of approximately $6 \mathrm{ohms}$. With a direct current of 1 ampere in the windings, the reactance of the inductor $L$ to 0.1

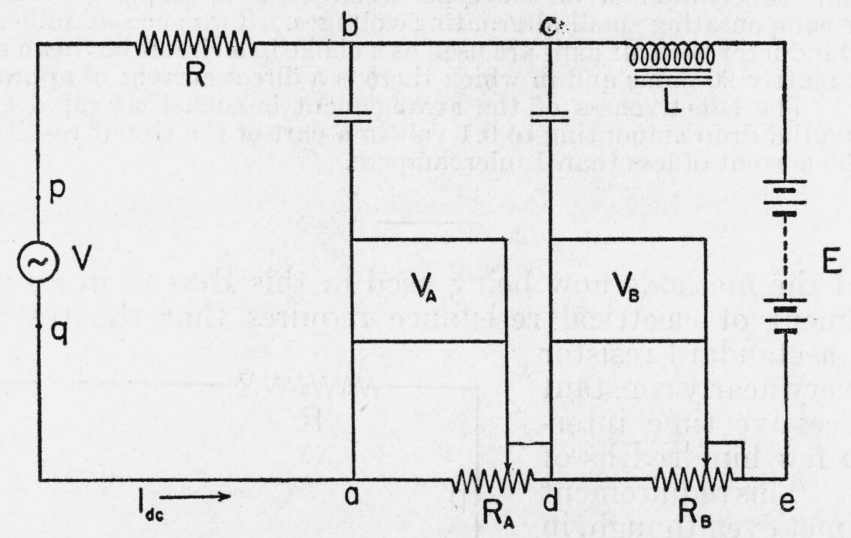

FIGURE 2. Simplified circuit of figure 1 shown with the additions of the inductor, $L$, and the vacuum-tube amplifiers, $V_{A}$ and $V_{B}$, which taken as $a$ whole serve as $a$ choke to the pulsating potential difference.

volt at the fundamental frequency of approximately 45 cycles per second is somewhat in excess of 1,000 ohms.

$V_{A}$ and $V_{B}$ are resistance-coupled, phase-inverter, vacuum-tube amplifiers adjusted very nearly to a 1 to 1 voltage amplification. Consequently, sudden and cyclic changes in their input voltage cause very nearly equal and opposite changes in the potential drops in their load resistors, $R_{A}$ and $R_{B}$.

Referring to figure 2, since the reactance of $L$ is much larger than the a-c resistance of $R, R_{A}$, and $R_{B}$, the small fluctuating voltage, $V$, between $p$ and $q$ is impressed on amplifier $V_{A}$ between $a$ and $b$. A fluctuating potential difference of approximately the same magnitude but opposite in phase appears across output resistor $R_{A}$ of amplifier $V_{A}$. This output potential drop between $a$ and $d$ will largely compensate the input voltage across $a$ and $b$; and the uncompensated voltage, which was found to be approximately equal to $v / 20$, will appear across $c$ and $d$. This uncompensated voltage serves as the input voltage for amplifier $V_{B}$ and is largely compensated by the output potential drop across $d$ and $e$. The resultant uncompensated voltage between $c$ and $e$ 
is approximately equal to $v /(20 \times 20)$, or $v / 400$. The large reactance of the inductor, $L$, prevents this small voltage from changing the test current by as much as 1 microampere.

Details of one of the vacuum-tube amplifiers used as an alternatingvoltage compensator are shown in figure 3 . The values for the resistors, capacitors, and biasing voltages were selected experimentally. With an input voltage of 0.1 volt between $a$ and $b$, output resistor $R_{A}$ is adjusted until a minimum voltage between $c$ and $d$ is indicated by a vacuum-tube voltmeter. This indicated minimum will not be zero, since there is some distortion in the voltage compensator. Furthermore, this distortion depends to some extent on the characteristics of the tubes, since the indicated minimum was found to be

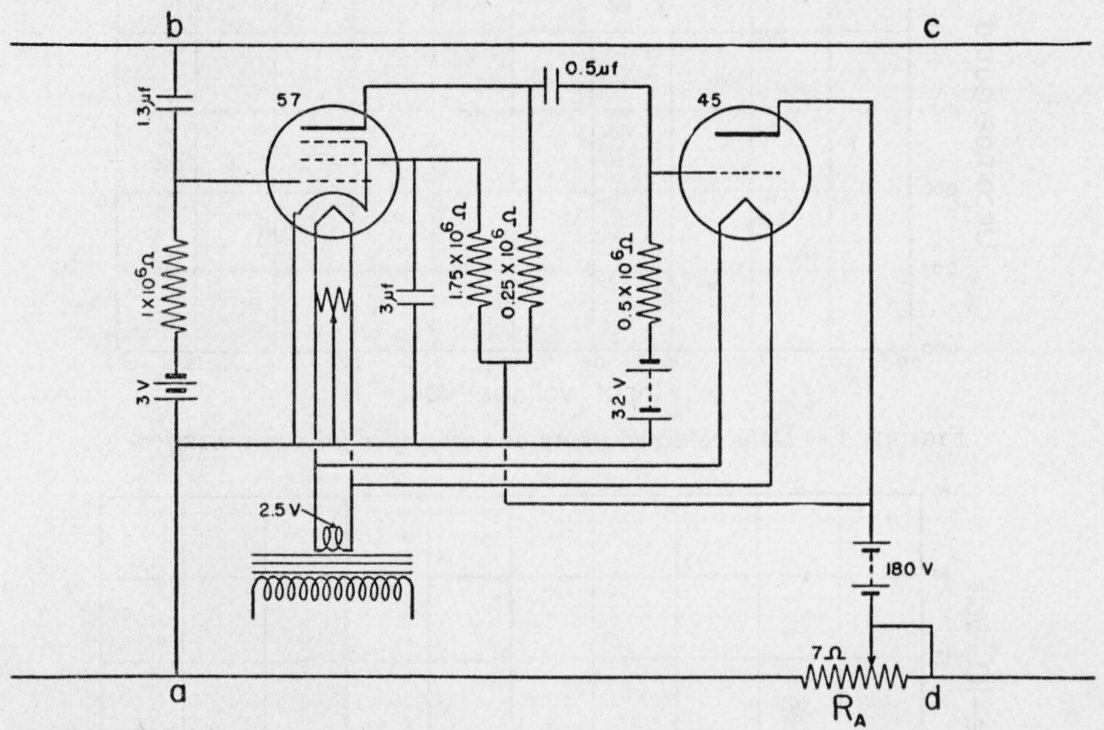

FIGURE 3. Details of a vacuum-tube amplifier used for compensating pulsating potential differences.

different on changing a tube for another of the same type number and then making the best obtainable adjustment.

The performance characteristics of the alternating-voltage compensators for voltages of different frequencies are shown in figures 4 and 5 . These data were obtained with a direct current, $I_{d c}$, of 0.7 ampere in resistor $R$. However, the performance of the amplifiers will not be appreciably affected if the magnitude of $I_{d c}$ is changed to a different value, provided that the final adjustments of the output resistors $R_{\mathrm{A}}$ and $R_{\mathrm{B}}$ are made when the direct current in $R$ has the desired magnitude.

The data shown in figure 4 were obtained using only the amplifier $V_{\mathrm{A}}$ for compensation. It will be seen from figures 2 and 4 that when the input alternating voltage between $a$ and $b$ is 0.1 volt, the uncompensated voltage between $b$ and $d$ is approximately 0.005 volt. In this case, using only one amplifier, the input voltage has been compensated within approximately 5 percent. 
The data shown in figure 5 were obtained using both amplifiers for compensation. It will be seen from figures 2 and 5 that when the alternating input voltage between $a$ and $b$ is less than 0.1 volt the un-

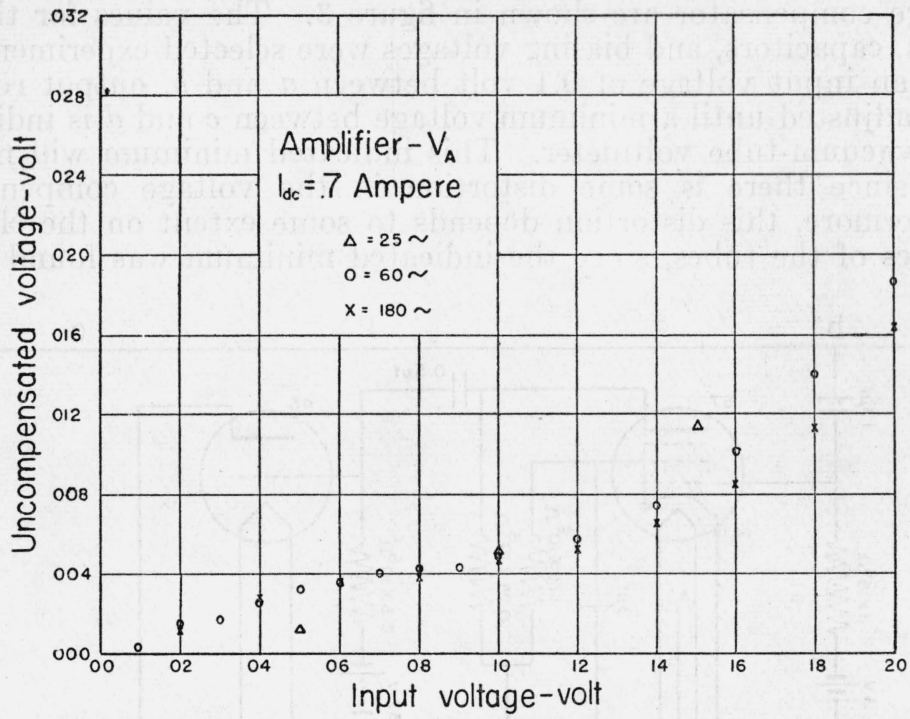

FIGURE 4.-Data obtained using one amplifier for compensation.

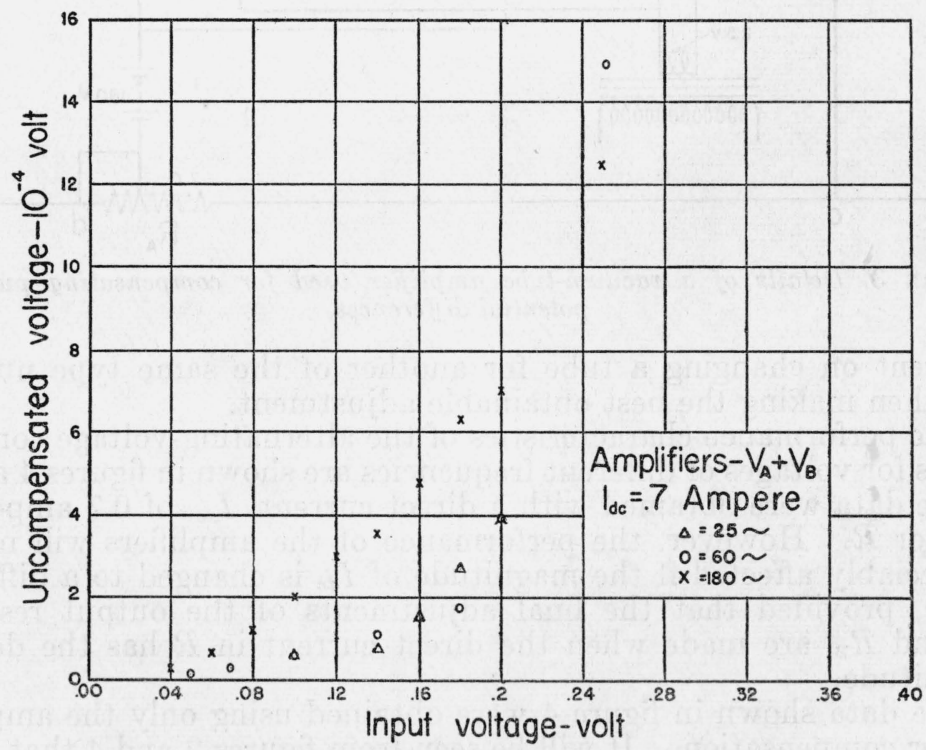

Figure 5.-Data obtained using two amplifiers for compensation.

compensated voltage between $c$ and $e$ is less than 0.0002 volt for frequencies between 25 and 180 cycles per second.

As the initial impedance of the inductor at the fundamental frequency of 45 cycles per second is approximately $1,000 \mathrm{ohms}$, the 
fluctuating voltage of 0.0002 volt between $c$ and $e$ of figure 2 causes fluctuations in the test current through the battery of approximately $2 \times 10^{-7}$ ampere. However, the alternating component of the current in $R$ is larger by approximately $1 \times 10^{-7}$ ampere as a result of the alternating component of the current in the grid resistor of the 57 tube. By the use of the two alternating-voltage compensators and the iron-cored inductor, it has been possible to limit the alternating component of the current in $R$ to less than one part in a million of the direct current.

Washington, April 18, 1940. 